\title{
Air Traffic Controller Alertness Level Detection
}

\author{
Aziz Parmaksız and Dr. Mehmet Göktürk \\ Department of Computer Engineering, Gebze Technical University, Kocaeli, Turkey
}

\begin{abstract}
Air traffic controllers (ATC) are responsible for safely take off airplanes, ensuring aircraft flight levels, maintain the safe distance between the aircrafts, and safely landing aircraft. Controllers must be fresh and be able to respond quickly to events, as there are numerous sudden decisions in their daily work.

The aim of this study is to demonstrate the stress levels of the ATC using the Galvanic Skin Response (GSR) and Heart Rate (HR) sensors by the hybrid method. As a result of experimental study with 20 subjects, it was seen that the classification success of the stressed condition was obtained with the Naive Bayes Multinomial method proposed by $91 \%$.
\end{abstract}

Keywords: ATC, stress, GSR, HR.

\section{Introduction}

Air traffic controllers are people trained to maintain the safe, orderly, and expeditious flow of air traffic in the global air traffic control system. ATC have to make countless sudden decisions within a short period of time to ensure flight safety and flow of traffic in a daily basis. With increased air traffic, the workload of the controllers has also increased. This responsibility and workloads make ATC naturally stressful.Stress is a physiological response to the mental, emotional, and physical challenges user faced.

This response triggers the autonomic nervous system, and putting the body in a mode of taking precautions against them. The intensity of the stress level shifts the body to panic state and making authority is adversely affected.ATC, responsible for air traffic and human safety, must be alert to all situations that can occur at any moment. This can be achieved by their alertness levels being high. The measurement of the emotional states of the controllers is not sufficient in terms of the continuity of air traffic management. The determination of the level of stress and the determination of the alertness levels of the controllers according to these stress levels will help avoiding possible problems in the air traffic function.

\section{Literature Works}

There are many studies in the literature about identifying whether people are stressed or not. The GSR sensor is the most commonly used sensor to identify the stress state [2, 4, 5, 8, 9, and 11]. GSR also known as Skin Conductance (SC) or Electro Dermal Response (EDR), that skin resistance is measured by electrodes placed on two fingers. When being stressed body produce sweat and that sweat increase electrical conductivity. When skin electrical conductivity increases, conversely skin electrical resistance decreases.

Changes in the heart rhythm are another method used to determine stress. The experiment in [7] authors give a coin-stacking task to stimulus and observe HR frequency changes. They improved changes in LF/HF ratio affected the mental status of the subjects and increased their sympathetic nerves activity.

\section{Method}

\subsection{Collecting Data}

In this experiment, the GSR sensor and ear clipped heart rate sensor, which can work with Ardino Uno development cards, were used. 
The Ardino Uno development card connected to the portable computer is fed with 5V electrical voltage. The GSR value obtained here shows the galvanic skin resistance. The skin electrical conductivity value is siemens (S) value and the skin electrical resistance value is read as ohm (R). In the event of stress, the sweat increases the flow through the GSR electrodes connected to the fingers, because of creating a salty pervious layer on the skin. The increasing current is inversely proportional to the falling of the skin electrical resistance. This indicates that the skin's electrical resistance value is inversely proportional to the stress level.

\subsection{Test Protocol}

Experiments done with 20 (9 female, 11 male) controllers worked at the Turkish Air Traffic Control Center. Before experiment each controlled informed about experiment, and agree with using their results. The working experience of the controllers varies from 6 months to 19 years. The age range of males is between 26 and 35 and females are between 26 and 42.7 of participants use cigarettes. In this study, the responses to the workload of the controllers and the possible traffic conflict situations were measured in the real environment and in the CWP simulation environment. Both environments have exactly same user interface and HMI. The sensor values were collected while controllers on CWP board, out of CWP board and on CWP simulation environment.

In simulation environment each of 10 participants has a 6 month controller experience was asked to control traffics in the approach sector on the CWP board for 35 minutes. In a different room, 4 controller take pseudo pilot positions and implements instructions ordered from voice communication system. At the beginning, controller has 1 traffic in the field of responsibility as time progressed, the number of traffic increased to 36 when it reached 20 minutes. During the experiments conflicts were consciously created at 10th and 20th minutes, to monitor the stress level of controller at that moment.

In the real CWP environment, 10 minutes sensor values were collected from the subjects with the experience of controlling between 1 and 19 years. During this time, participants were asked to do their routine work. At the moment of operation, the number of traffic within the responsibility of the controllers varies between 12 and 35. Participants were checking transit flights and generally making traffic separation. In this environment, the events that can cause the stresses and time are recorded by monitoring the operation of the controllers.

4 minute sensor values were collected from the subjects out of real and simulation CWP board, to obtain the normal GSR and HR values of the subjects.

\subsection{Experiment Result}

As a result of the experiment the average values of GSR 335 and HR 85 were obtained while controllers at out of simulation and real CWP environment. In the simulation environment, participants' GSR values were observed to fall below the initial level due to increased traffic and intensity. For simulation environment these values measured as 100 GSR and $98 \mathrm{HR}$, respectively. In the real CWP environment average of $230 \mathrm{GSR}$ and $80 \mathrm{HR}$ were measured.

32 data sets collected from this experiment. To verify the different tests, we used the WEKA learning machine for testing and applied cross validation by choosing $\mathrm{k}$ fold number 10 . We have obtained good results with Naive Bayes Multinomial methods as $91 \%$ success. We obtain $84 \%$ of success while ignoring HR values.

TABLE I : Classification Table

\begin{tabular}{lll}
\hline \hline GSR & HR & CLASS \\
\hline 353 & 64 & not stressed \\
103 & 86 & stressed \\
202 & 79 & not stressed \\
116 & 104 & stressed \\
195 & 88 & stressed \\
307 & 95 & not stressed \\
418 & 73 & not stressed \\
353 & 64 & not stressed \\
103 & 86 & stressed \\
$\ldots$ & $\ldots$ & $\ldots$ \\
\hline \hline
\end{tabular}




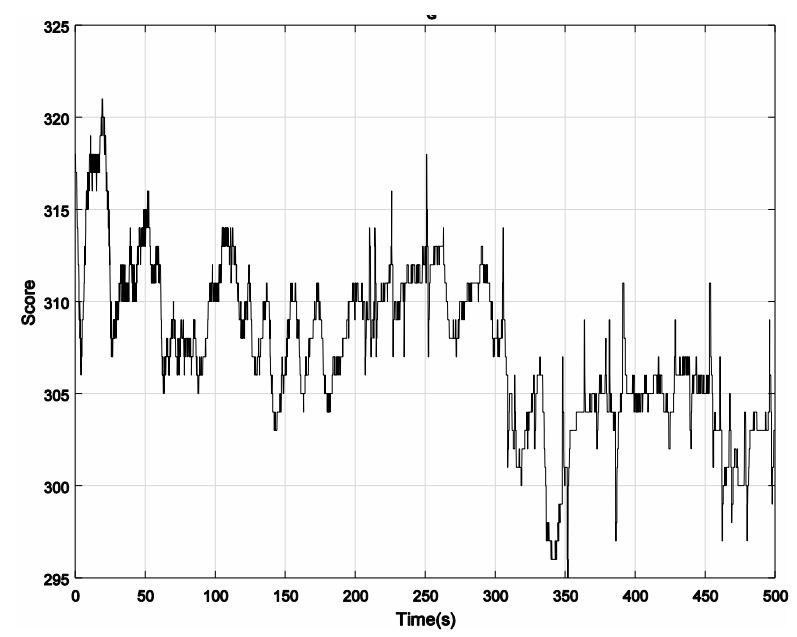

Fig. 1: User-1 GSR values on CWP Board

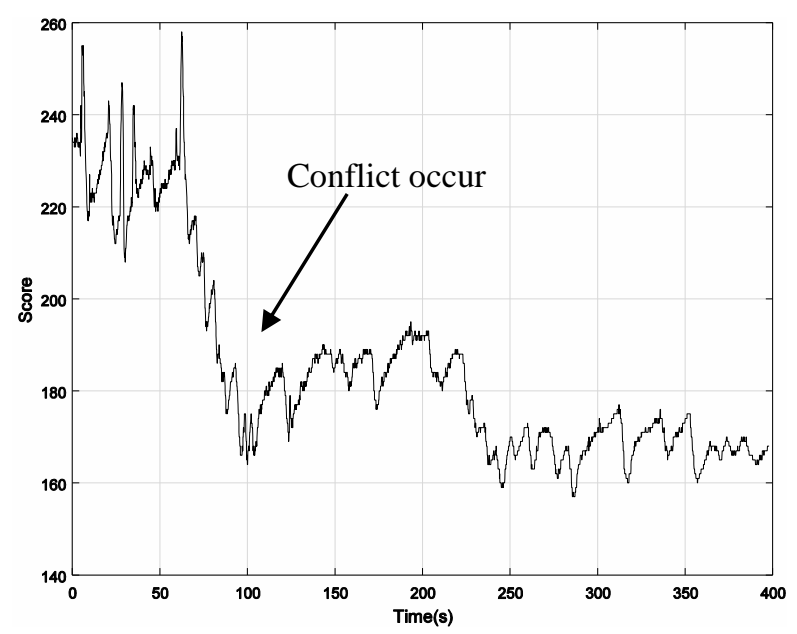

Fig. 3: User-4 GSR values when conflict occur at real CWP Environment

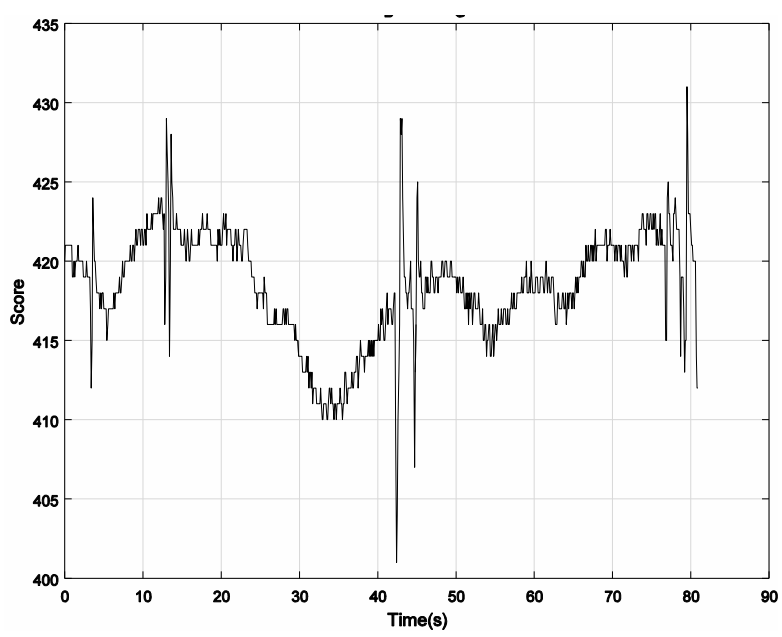

Fig. 2: User-1 GSR values while relaxing

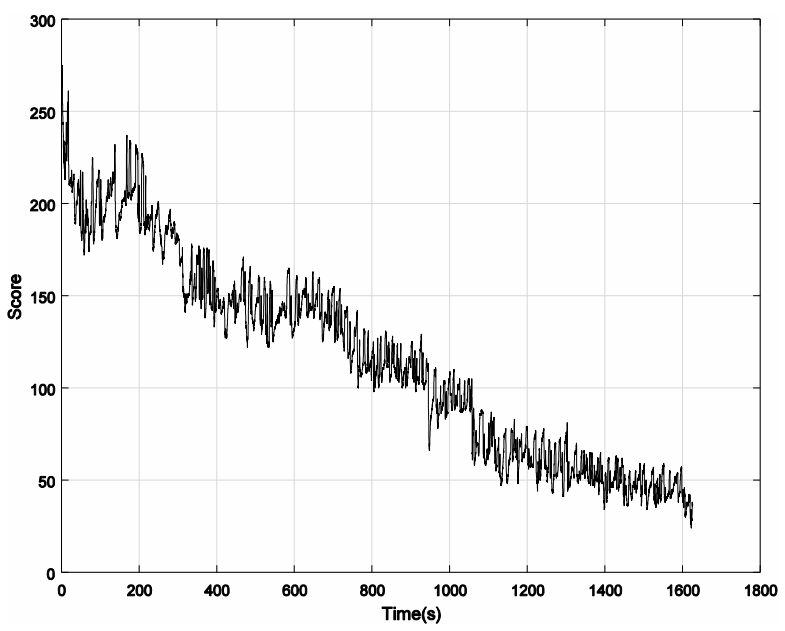

Fig. 4: User-11 GSR values on Simulation Environment

\section{Result}

The GSR values are more sensitive to events in the stress detection of ATC. That makes it possible to use the GSR sensor as a priority sensor for stress definition, and HR sensor as a confirmatory factor in stress detection.

The HR values were obtained in 20 seconds period. That time period good enough for an aircraft to collapsed or lost. For an ATC every one seconds is important. So with GSR sensor stress changes can obtain faster. The variety of GSR value in 2 seconds show us how GSR sensor sensitive to event. That is the second reason why GSR sensor must be used as priority sensor for detecting stress of ATC.

In the real environment, the GSR values at the CWP board from beginning to end were at the same level unless conflict occurs, but this value tended to decrease in the simulation environment. When the two situations are compared, we can say that in the simulation environment the stress level of the participant increases with the increasing workload. Work experience is another factor. We see that GSR values of controllers, who have over 10 years of experience and control same amount of traffic, flow in same level.

\section{Acknowledgment}

Special thanks to Turkish Air Traffic Control Center for improving this experiment. 


\section{Reference}

[1] http://en.wikipedia.org/wiki/Alertness

[2] Coghlan, Niall, and R. Benjamin Knapp. "Sensory Chairs: A system for biosignalresearch and performance." (2008).

[3] Levenson, Robert W., et al. "Emotion and autonomic nervous system activity in the Minangkabau of West Sumatra." Journal of personality and social psychology 62.6 (1992): 972.

[4] Sun, Feng-Tso, et al. "Activity-aware mental stress detection using physiological sensors." International Conference on Mobile Computing, Applications, and Services. Springer Berlin Heidelberg, 2010.

[5] Villarejo, MaríaViqueira, BegoñaGarcíaZapirain, and AmaiaMéndez Zorrilla. "A stress sensor based on Galvanic Skin Response (GSR) controlled by ZigBee."Sensors12.5 (2012): 6075-6101.

[6] Papathanasiou, George, et al. "Effects of smoking on heart rate at rest and during exercise, and on heart rate recovery, in young adults." Hellenic J Cardiol54.3 (2013): 168-177.

[7] Seong, H. M., Lee, J. S., Kim, W. S., Lee, H. S., Youn, Y. R., \& Shin, T. M. (2004). The analysis of mental stress using time-frequency analysis of heart rate variability signal. Journal of Biomedical Engineering Research, 25(6), 581-587.

[8] Sandulescu, V., Andrews, S., Ellis, D., Bellotto, N., \& Mozos, O. M. (2015, June). Stress detection using wearable physiological sensors. In International Work-Conference on the Interplay Between Natural and Artificial Computation (pp. 526-532). Springer International Publishing. https://doi.org/10.1007/978-3-319-18914-7_55

[9] Mozos, Oscar Martinez, et al. "Stress detection using wearable physiological and sociometric sensors." International Journal of Neural Systems 27.02 (2017): 1650041.

[10] ROWE, Dennis W.; SIBERT, John; IRWIN, Don. Heart rate variability: Indicator of user state as an aid to humancomputer interaction. In: Proceedings of the SIGCHI conference on Human factors in computing systems. ACM Press/Addison-Wesley Publishing Co., 1998. p. 480-487.

[11] Wijsman, Jacqueline, et al. "Towards mental stress detection using wearable physiological sensors." Engineering in Medicine and Biology Society, EMBC, 2011 Annual International Conference of the IEEE. IEEE, 2011.

https://doi.org/10.1109/iembs.2011.6090512

[12] Salahuddin, Lizawati, et al. "Ultra short term analysis of heart rate variability for monitoring mental stress in mobile settings." Engineering in Medicine and Biology Society, 2007. EMBS 2007. 29th Annual International Conference of the IEEE. IEEE, 2007.

https://doi.org/10.1109/iembs.2007.4353378 